\title{
Adaptación de la escala Creative Environment Perceptions al español*
}

\section{Adaptation of the Creative Environment Perceptions Scale into Spanish}

Recibido: julio 4 de 2013 | Revisado: mayo 17 de 2014 | Aceptado: mayo 17 de 2014

\author{
JOAN BOADA-GRAU ** \\ ANDREU VIGIL-COLET **** \\ Universitat Rovira i Virgili, Tarragona, España \\ José-CARLOS SÁNCHEZ-GARCÍA **** \\ Universidad de Salamanca, España \\ Aldo-Javier PrIZMIC-KuZMICA ****** \\ Escuela de Alta Dirección y Administración, \\ Barcelona, España
}

doi.org/10.11144/Javeriana.UPSY13-4.aece

Para citar este artículo: Boada-Grau, J., Vigil-Colet, A., Sánchez-García, J. -C., \& Prizmic-Kuzmica, A. -J. (2014). Adaptación de la escala Creative Environment Perceptions al español. Universitas Psychologica, 13(4), 1357-1366. http://dx.doi.org/10.11144/ Javeriana.UPSY13-4.aece

* Artículo de investigación

** Profesor Titular de Universidad. Correo electrónico: joan.boada@urv.cat

**** Catedrático de Universidad. Conflicto de intereses CI POLICY. Correo electrónico: andreu.vigil@ urv.cat

****** Profesor Titular de Universidad. Correo electrónico: jsanchez@usal.es

${ }_{* * * * * *}^{*}$ Profesor. Correo electrónico: jprizmic@eada.edu

\section{RESUMEN}

Este estudio se enmarca dentro de la propuesta de Mayfield y Mayfield (2008, 2010) sobre las percepciones del entorno creativo. El objetivo fue realizar la adaptación al español de la escala Creative Environment Perceptions de nueve ítems y analizar sus propiedades psicométricas. Los participantes fueron 975 empleados españoles ( $48.5 \%$ hombres y $51.5 \%$ mujeres). Los resultados obtenidos demuestran, después de realizar análisis confirmatorio, una estructura constituida por tres factores: Apoyo a la Creatividad, Características del Trabajo y Bloqueos a la Creatividad. Además, los tres factores obtenidos tienen una fiabilidad adecuada e igualmente se constatan indicios de validez, si se toman como referencia algunos correlatos externos y algunas escalas que hacen referencia a la adicción al trabajo, el burnout y el potencial creativo. En suma, la presente escala puede resultar idónea para identificar de manera apropiada los tres factores.

Palabras clave

Percepciones del entorno creativo; creatividad; adaptación; escala; estudio instrumental

\section{A B S T R A C T}

This study falls within the framework of Mayfield and Mayfield's proposal $(2008,2010)$ on creative environment perceptions. The objective was to adapt the 9-item Creative Environment Perceptions Scale into Spanish and analyse its psychometric properties. The sample for the present study was made up of 975 Spanish employees (48.5\% men and 51.5\% women). After carrying out a confirmatory analysis the results displayed a three factor structure: Support to creativity, Job characteristics and Blocks to creativity. Furthermore, the three factors showed appropriate reliability and some signs of validity were also found when they were contrasted with certain external correlates and scales that refer to workaholism, burnout and creative potential. By way of summing up, the present scale may prove ideal for appropriately identifying support to creativity, job characteristics and blocks to creativity.

Keywords

Creative environment perceptions; creativity; adaptation; scale; study instrument 
El entorno socioeconómico actual exige a las organizaciones y a las empresas adaptarse constantemente para ser sostenibles (Mumford, 2012). Una organización innova y crea si motiva a los empleados; para ello, es necesario que les proporcione todo tipo de recursos y que el ambiente de trabajo sea gestionado de forma adecuada (Amabile, 1988). Los recursos necesarios para la innovación son los financieros, los materiales y de información, la generación de oportunidades y el tiempo para explorar nuevas formas de hacer las tareas (Amabile, 1997).

La creatividad puede ser potenciada por ciertas prácticas directivas como la incentivación de la autonomía y la retroalimentación, la de proponer tareas y metas intelectualmente desafiantes y la de constituir equipos de trabajo con climas innovadores (Boada-Grau, De-Diego, De-Llanos-Serra, \& Vigil-Colet, 2011) de acuerdo a las habilidades y personalidades de los empleados y mediante la potenciación del pensamiento flexible y comportamientos persistentes (De Dreu, Baas, \& Nijstad, 2008).

Además, diversas investigaciones consideran que el ambiente creativo (y en consecuencia la creatividad) se ve potenciado por el estilo de liderazgo que practican los directivos (Amabile, Schatzel, Moneta, \& Kramer, 2004; Mayfield \& Mayfield, 2005, 2007). Los directivos y los mánager han de considerar la creatividad como un objetivo importante que debe ser alimentado y facilitado (Mueller, Melwani, \& Goncalo, 2012).

La investigación en la innovación organizacional se ha basado esencialmente, entre otros aspectos, en las percepciones de los empleados sobre la creatividad en el trabajo (Mayfield \& Mayfield, 2008). Algunas corrientes de investigación indican que el trabajo creativo aparece cuando las tareas presentadas conllevan problemas complejos y mal definidos, donde la obtención de los resultados precisa la generación de soluciones novedosas y útiles (Mumford, Scott, Gaddis, \& Strange, 2002). Igualmente importante es que una de las medidas utilizadas para capturar el potencial imaginativo de los empleados se realice a través de sus percepciones sobre el ambiente creativo (Amabile, 1993, 1996; ) que se dan en un contexto laboral. El contexto incluye los elementos tanto proximales (por ejemplo, el diseño del trabajo) como distales (por ejemplo, comportamientos de gestión y directivos, políticas de la organización, etc.) (Shalley, Gilson, \& Blum, 2000). Desde una perspectiva psicosocial, los outputs innovadores siempre surgen de un contexto (Mayfield \& Mayfield, 2008). Así, las interacciones que se realizan entre los contextos de trabajo y los empleados fomentan o inhiben la expresión creativa de estos últimos (Amabile, 1998; Amabile, Hadley, \& Kramer, 2002; McLean, 2005).

Varios autores consideran que la percepción de un entorno creativo puede mejorarse a través de un diseño apropiado de la organización (Amabile, 1993; Forbes \& Domm, 2004; Shalley et al., 2000). Y a su vez, estas percepciones al ser mejoradas permiten aumentar la creatividad del trabajador (Amabile, 1993, 1997, 1998; Amabile et al., 1996; Bryant, 2003; Stoker, Looise, Fisscher, \& De Jong, 2001; Williams, 2001).

En una investigación, Von Krogh (1998) examinó el intercambio de conocimientos tácitos entre trabajadores, llegando a la conclusión de que la calidad de información de este intercambio es un componente clave en la creatividad; los intercambios se operan en las organizaciones que facilitan el ambiente creativo, este tiene efectos en el trabajador potenciando la creatividad. Además, se identifican algunos factores fundamentales para que aparezca la creatividad como el soporte que la organización ofrece a la creatividad del trabajador, la naturaleza creativa del trabajo en sí y los bloqueos de la creatividad derivados de la organización y el diseño del trabajo.

Basándose en la discusión que precede, Mayfield y Mayfield $(2008,2010)$ diseñaron y desarrollaron una escala sobre las percepciones del entorno creativo que tiene tres componentes: el Apoyo a la Creatividad, las Características de Trabajo y los Bloqueos de la Creatividad. El objetivo de la presente investigación ha sido realizar una adaptación al español del original inglés. Así, se ha analizado la estructura interna (análisis factorial confirmatorio), la fiabilidad y la aportación de índices de validez convergente de la escala investigada. 


\section{Método}

\section{Participantes}

La muestra heterogénea estuvo compuesta por 975 empleados de España (48.5\% hombres y 51.5\% mujeres) y la edad media es de 42.49 años ( $D E=$ 11.25). El estado civil es: casado (60.8\%), pareja de hecho (6.9\%), soltero (23.8\%), divorciado/ separado (7.5 \%) y viudo (1.1\%). El nivel formación académica se distribuye así: sin certificado o título académico el $1.4 \%$, estudios primarios acabados el 22.6\%, estudios secundarios acabados el 39.1\%, diplomatura o ingeniero técnico el $18.4 \%$, licenciado, ingeniero superior o arquitecto el $12.6 \%$ y máster/doctorado el $6 \%$.

\section{Instrumentos}

La escala de medida Creative Environment Perceptions ([CEP]; Mayfield \& Mayfield, 2010) está compuesta por nueve ítems en la versión inglesa. El anclaje de respuesta es de cinco puntos (de 1 $=$ Completamente en desacuerdo a $5=$ Completamente de acuerdo). Dicha versión fue traducida al español (véase Apéndice) de acuerdo tanto al método de traducción y retrotraducción (backtranslation) (Hambleton, Merenda, \& Spielberger, 2005; Muñiz \& Bartram, 2007) como a los criterios establecidos para adaptar un instrumento de medida de una cultura a otra (Balluerka, Gorostiaga, Alonso, \& Haranburu, 2007). Las escalas originales son tres: Soporte a la Creatividad $(\alpha=$ 0.85; por ejemplo, "2.- Mi grupo de trabajo apoya las nuevas maneras de hacer las cosas"); Características del Trabajo ( $\alpha=0.71$; por ejemplo, "5.-Mi trabajo es retador") y Bloqueos a la Creatividad ( $\alpha$ = 0.81; por ejemplo, "8. - Las políticas de mi organización impiden la espontaneidad en el trabajo”).

La escala Creative Potential and Practised Creativity ([CPPC]; DiLiello \& Houghton, 2008) está compuesta de 17 ítems en la versión inglesa. La versión española la realizaron Boada-Grau, SánchezGarcía, Prizmic-Kuzmica y Vigil-Colet (2013). Se responde en un formato de cinco puntos (desde 1 = Completamente en desacuerdo hasta $5=$ Com- pletamente de acuerdo). Los ítems están agrupados en tres subescalas: Potencial Creativo tiene 6 ítems ( $\alpha=0.84$; por ejemplo, "2.- Tengo confianza en mi capacidad para solucionar problemas de forma creativa"), Creatividad Práctica está formado por 5 ítems ( $\alpha=0.84$; por ejemplo, "8.- En el trabajo me invitan a que presente ideas de mejora") y Percepción de Apoyo Organizacional conformada por 6 ítems ( $\alpha=0.94$; por ejemplo, "14.- En mi organización se anima a la gente a resolver los problemas de forma creativa").

La escala de Adicción al Trabajo ([WorkBAT]; Burke, Richardsen, \& Martinussen, 2002; McMillan, Brady, O’Driscoll, \& Marsh, 2002), en la versión española realizada por Boada-Grau, Prizmic-Kuzmica, Serrano-Fernández y Vigil-Colet (en prensa) tiene 19 ítems y 2 subescalas. El formato de respuesta es de cinco opciones (De $1=$ Nada de acuerdo a $5=$ Totalmente de acuerdo). La primera subescala se denomina D (Driven) está integrada por 12 ítems ( $\alpha=$ 0.82; por ejemplo, "4.- A menudo desearía no estar tan comprometido con mi trabajo"), la segunda se llama J (Work Enjoyment) compuesta por 7 ítems ( $\alpha=0.83$; por ejemplo, "5.- Mi trabajo es tan interesante que a menudo no parece trabajo").

La escala Dutch Work Addiction Scale ([DUWAS]; Schaufeli, Shimazu, \& Taris, 2009), en la versión española (Del-Líbano, Llorens, Salanova, \& Schaufeli, 2010) consta de una estructura bifactorial y 10 ítems. El anclaje de las respuestas es de cuatro puntos como: $1=($ Casi) Nunca, $2=$ A veces, $3=$ A menudo y $4=$ (Casi) Siempre. Los dos factores tienen cinco ítems cada uno. El primero se denomina Trabajar Excesivamente (por ejemplo, "3.- Siempre parece que voy con prisas y a contrarreloj") y el segundo Trabajar Compulsivamente (por ejemplo, "14.- Me siento obligado/a a trabajar duro, incluso cuando no lo estoy disfrutando"). Las fiabilidades son de 0.75 y 0.81 , respectivamente.

La versión española del Maslach Burnout Inventory-General Survey ([MBI-GS]; Salanova, Schaufeli, Llorens, Peiró, \& Grau, 2000; Schaufeli, Leiter, Maslach, \& Jackson, 1996) consta de 15 ítems y tres subescalas. El anclaje de respuestas es de 6 puntos (desde ninguna vez hasta todos los días). La de Eficacia Profesional comprende 6 ítems $(\alpha=0.78$; 
por ejemplo, "10.- En mi opinión soy bueno en mi puesto"), la de Cinismo tiene 5 ítems $(\alpha=0.85$; por ejemplo, "13.- Me he vuelto más cínico respecto a la utilidad de mi trabajo") y la de Agotamiento comprende 5 ítems $(\alpha=0.87$; por ejemplo, "2.Estoy 'consumido' al final de un día de trabajo”).

Finalmente, para evaluar la validez convergente también se utilizaron algunos correlatos denominados indicadores externos (Boada-Grau, SánchezGarcía, Prizmic- Kuzmica, \& Vigil-Colet, 2012; Boada-Grau, Prizmic-Kuzmica, González-Fernández, \& Vigil-Colet, 2013) en forma de cuestiones que los informantes debían de contestar utilizando frecuencias. Así, se les hacían preguntas como: "En términos generales ite sientes saludable?", "Teniendo en cuenta la felicidad icómo estás de feliz con tu vida?", "iCon que frecuencia te llevas trabajo a casa?”, “iEstás obligado/presionado en tu organización a dar el conocimiento que tú tienes a ella?" y "iEstás obligado/presionado en tu organización a proporcionarle propuestas innovadoras para mejorar?".

\section{Procedimiento}

Los datos se recogieron a partir de los cuestionarios con el consentimiento de los responsables de las empresas y de los mismos empleados. Las escalas se administraron en horario laboral por parte de entrevistadores previamente entrenados a tal efecto, que les dieron las instrucciones oportunas para contestar las escalas. Se les solicitó a los participantes que fueran sinceros en sus contestaciones. La participación fue voluntaria y confidencial. Se garantizó el anonimato de las respuestas. Los participantes y las organizaciones en las que se llevó a cabo la administración de los cuestionarios fueron elegidos por accesibilidad. Para la obtención de los participantes, se utilizó el muestreo no probabilístico, también denominado aleatorio-accidental (Kerlinger, 2001).

\section{Análisis de datos}

Se utilizó el Mplus (Versión 6.12) para realizar un análisis factorial confirmatorio, obteniendo una estructura latente de tipo trifactorial: (1) Soporte a la Creatividad, (2) Características del Trabajo y (3) Bloqueos a la Creatividad. El modelo de ecuaciones estructurales (SEM) tiene ventajas para ensayar las propiedades de una escala, así proporciona un método para examinar la estructura subyacente de las variables latentes, que son factores que no pueden medirse directamente, sino que pueden estimarse mediante otras variables manifiestas (Joreskog, 1993; Schumacker \& Lomax, 1996). Además, se utilizó el programa SPSS 19.0 para llevar a cabo los siguientes análisis: (a) el análisis de la fiabilidad de los tres factores mediante el coeficiente alfa de Cronbach, (b) las correlaciones entre los tres factores constituyentes de la escala y (c) la correlación de los tres factores de la escala con otras medidas como la creatividad (CPPC-17), la adicción al trabajo (WorkBAT y DUWAS), la irritación, el burnout (MBI-GS), la personalidad (OPERAS) y varios correlatos externos.

\section{Resultados}

El AFC llevado a cabo sobre el CEP-9 mostró un buen ajuste el modelo de tres factores propuesto inicialmente (original en lengua inglesa). En la presente investigación, se utilizaron los siguientes indicadores de bondad de ajuste: TLI (Tucker-Lewis Index), CFI (Comparative Fit Index), RMSEA (Root Mean Square Error of Approximation). Aunque no hay unanimidad sobre los valores óptimos para los puntos de corte en los índices de ajuste de los modelos estructurales, existe un cierto consenso en que los valores iguales o superiores a 0.9 en el índice de Tucker-Lewis y índice de ajuste comparativo (CFI) son aceptables, considerándose excelentes cuando superan el valor de 0.95 (Lévy-Mangin \& Varela-Mallou, 2006). Por otra parte, la raíz cuadrada media de error de aproximación (RMSEA) se considera aceptable cuando es inferior a 0.08 y excelente cuando es igual o inferior a 0.05 (Bentler, 1990; Hu \& Bentler, 1999; Fan \& Sivo, 2007). En la Figura 1 se muestran los siguientes indicadores $(\mathrm{TLI}=0.98 ; \mathrm{CFI}=0.98 ; \mathrm{RMSEA}=0.03)$, estos indican un ajuste aceptable del modelo, situándose todos los índices cercanos a los valores considerados 


$\begin{array}{cccc} & 0.81 & \text { ÍTEM 1 } & 0.33 \\ \text { F1.-Apoyo Creatividad } & 0.74 & \text { ÍTEM 2 } & 0.44 \\ & 0.91 & \text { ÍTEM 3 } & 0.17 \\ & 0.67 & & \\ & -0.49 & & \\ \text { F2.-Características Trabajo } & 0.41 & \text { ÍTEM 4 } & 0.82 \\ & 0.66 & \text { ÍTEM 5 } & 0.55 \\ & 0.47 & \text { ÍTEM 6 } & 0.77 \\ \text { F3.-Bloqueos Creatividad } & -0.51 & & \\ & 0.8 & \text { ÍTEM 7 } & 0.35 \\ & 0.89 & \text { ÍTEM 8 } & 0.19 \\ & 0.57 & \text { ÍTEM 9 } & 0.67\end{array}$

Figura 1. Análisis factorial confirmatorio de la escala CEP-9 $\left(\chi^{2}=41.165 ; g l=24 ;\right.$ P-valor $=0.016 ; R M S E A=0.03$; $\mathrm{CFI}=0.98$ y $\mathrm{TLI}=0.98$ ).

Fuente: elaboración propia

aceptables. Además, todas las saturaciones oscilan entre 0.47 y 0.91 .

En la Tabla 1 se muestran la media, la desviación estándar, la fiabilidad, los intervalos de confianza, los correlatos externos y las correlaciones entre los tres factores de la escala CEP-9 y los factores de las escalas CPPC-17, WorkBAT, DUWAS y MBI. Los índices de validez se han obtenido correlacionando las subescalas del instrumento que se presentaron tanto con las escalas anteriores como con algunos criterios externos. En este sentido, se pueden observar algunas correlaciones significativas como las que se describen a continuación. Los tres factores de la escala, en general, correlacionan positivamente con cinco correlatos externos (sentirse saludable, felicidad, trabajo en casa, dar conocimiento y propuestas innovadoras). También se puede indicar que el Factor 1, el Factor 2 y el Factor 3 correlacionan positivamente con el potencial creativo, la creatividad practicada, la percepción de apoyo organizacional, la adicción al trabajo, la irritación cognitiva, la eficacia personal y la estabilidad emocional. Sin embargo, alguno de los tres factores presenta correlaciones negativas con la irritación emocional, el cinismo y el agotamiento. La fiabilidad oscila entre 0.71 y 0.85 , pudiendo incluso ser considerada como excelente si se tiene en cuenta el reducido número de ítems que componen cada uno de los tres. También, se muestran las correlaciones entre los factores que van de 0.33 a 0.44 .

\section{Discusión y conclusiones}

Los resultados de la investigación corroboran que la escala CEP-9 posee una estructura interna de tres factores, una fiabilidad adecuada y además unos índices de validez apropiados. El instrumento presentado evalúa el apoyo a la creatividad, las características del trabajo y los bloqueos organizacionales a la creatividad. Es la primera vez que la esta escala se presenta en una muestra de habla española. Los resultados del análisis factorial confirmatorio (AFC) del CEP-9, con una muestra española heterogénea, apoyan el modelo trifactorial de Mayfield y Mayfield (2010) con una muestra norteamericana de estudiantes (graduados y no graduados) en áreas de negocios con experiencia laboral.

Los resultados del análisis factorial confirmatorio de la presente escala apoyan el modelo de los tres factores y este muestra un ajuste satisfactorio. Todo ello se corrobora mediante los índices obtenidos dado que presentan un buen ajuste del modelo $(\mathrm{TLI}=0.98 ; \mathrm{CFI}=0.98 ; \mathrm{RMSEA}=0.03)$.

El primer factor tiene tres ítems (números: $1,2 \mathrm{y}$ 3), se denomina F1: Apoyo a la Creatividad y alude a que el superior, el grupo de trabajo y la organización animen al empleado a ser creativo. El segundo, que se llama F2: Características del Trabajo hace referencia a aspectos del trabajo como los recursos disponibles, el control sobre el trabajo desarrollado y si el trabajo es retador y está integrado por tres ítems 


\section{TABLA 1}

Factores de la escala CEP-9: estadísticos descriptivos, fiabilidad, intervalos de confianza y correlaciones con las variables sociodemográficas y los factores de las escalas (CPPC, WorkBAT, DUWAS y MBI-GS) y la matriz de correlaciones entre los tres factores de la escala analizada

\begin{tabular}{|c|c|c|c|c|}
\hline & & $\begin{array}{c}\text { F1 } \\
\text { Soporte a la } \\
\text { Creatividad }\end{array}$ & $\begin{array}{c}\text { F2 } \\
\text { Características } \\
\text { del Trabajo }\end{array}$ & $\begin{array}{c}\text { F3 } \\
\text { Bloqueos a la } \\
\text { Creatividad }\end{array}$ \\
\hline Media & & 9.65 & 11.29 & 9.52 \\
\hline $\mathrm{DE}$ & & 2.89 & 2.14 & 2.92 \\
\hline Fiabilidad & & 0.85 & 0.71 & 0.81 \\
\hline Intervalo de Confianza & & $0.83-0.87$ & $0.68-0.74$ & $0.79-0.83$ \\
\hline \multirow[t]{5}{*}{ Correlatos externos } & $\begin{array}{l}\text { En términos generales ite sientes saluda- } \\
\text { ble? }\end{array}$ & $0.09 * *$ & $0.14 * *$ & $0.08 * *$ \\
\hline & $\begin{array}{l}\text { Teniendo en cuenta la felicidad icómo } \\
\text { estás de feliz con tu vida? }\end{array}$ & 0.04 & $0.09 * *$ & 0.03 \\
\hline & $\begin{array}{l}\text { ¿Con qué frecuencia te llevas trabajo a } \\
\text { casa? }\end{array}$ & $0.17 * *$ & $0.18^{* *}$ & $0.2 * *$ \\
\hline & $\begin{array}{l}\text { ¿Estás obligado/presionado en tu organi- } \\
\text { zación a dar el conocimiento que tú tienes } \\
\text { a ella? }\end{array}$ & $0.07 *$ & $0.09 * *$ & -0.03 \\
\hline & $\begin{array}{l}\text { ¿Estás obligado/presionado en tu organiza- } \\
\text { ción a proporcionarle propuestas innova- } \\
\text { doras para mejorar? }\end{array}$ & $0.3 * *$ & $0.22 * *$ & $0.14 * *$ \\
\hline \multirow[t]{3}{*}{ СРPC } & Potencial Creativo & $0.28 * *$ & $0.25 * *$ & $0.1^{* *}$ \\
\hline & Creatividad Practicada & $0.68 * *$ & $0.47 * *$ & $0.34 * *$ \\
\hline & Percepción de Apoyo Organizacional & $0.75 * *$ & $0.5 * *$ & $0.45 * *$ \\
\hline \multirow[t]{2}{*}{ WorkBAT } & D-Driven & $0.14 * *$ & $0.23 * *$ & 0 \\
\hline & J-Work Enjoyment & $0.38 * *$ & $0.3 * *$ & $0.25 * *$ \\
\hline \multirow[t]{2}{*}{ DUWAS } & WkExcessive & $0.12^{*}$ & $0.17 * *$ & 0 \\
\hline & WkCompulsive & $0.1^{*}$ & $0.18 * *$ & 0.01 \\
\hline \multirow[t]{3}{*}{ MBI-GS 2vidadatividad } & Agotamiento & $-0.16 * *$ & $-0.16 * *$ & $-0.2 * *$ \\
\hline & Cinismo & $-0.35 * *$ & $-0.32 * *$ & $-0.29 * *$ \\
\hline & Eficacia personal & $0.35 * *$ & $0.37 * *$ & $0.21 * *$ \\
\hline F1 & & - & .- & -. \\
\hline $\mathrm{F} 2$ & & 0.44 & -. & -. \\
\hline F3 & & 0.41 & 0.33 & -. \\
\hline
\end{tabular}

$* * \mathrm{p}<0.01 ; * \mathrm{p}<0.05$

Fuente: elaboración propia

(números: 4, 5 y 6). Y el último es el F3: Bloqueos a la Creatividad constituido por tres ítems (números: 7,8 y 9) e indica las dificultades puestas por la organización para ser creativos. La fiabilidad aportada por la versión inglesa de Mayfield y Mayfield (2010) es aceptable para los tres factores dado que es de 0.82. En la versión española que se ha realizado en la presente investigación oscilan entre 0.71 y 0.85 .

En cuanto a los índices de validez podemos indicar que Mayfield y Mayfield (2010) no realizaron ningún estudio al respecto. No obstante, se han incluido algunas escalas de contraste y criterios externos. En general, los tres factores de la escala analizada correlacionan de forma significativa tanto con correlatos externos como con otras escalas. Aparecen algunas correlaciones negativas, estas son: el agotamiento con F1 $(r=-0.16, p<0.01)$, F2 $(r=-0.16, p<001)$ y F3 $(r=-0.2, p<0.01)$; y el cinismo con F1 $(r=-0.35, p<0.01)$, F2 $(r=$ $-0.32, p<0.01)$ y F3 $(r=-0.29, p<0.01)$. 
Sin embargo, en general los tres factores se asocian positivamente a los correlatos externos, por ejemplo, F1 se relaciona con el sentirse saludable $(r=0.09, p<0.01)$, F2 con la frecuencia de llevarse trabajo a casa $(r=0.18, p<0.01)$ y F3 con la proposición de ideas innovadoras $(r=$ $0.14, p<0.01)$. En cuanto a las escalas de contraste utilizadas se puede indicar que se observan correlaciones positivas de F1 con el CPPC (por ejemplo, Creatividad Practicada; $(r=0.68, p$ $<0.01$ ), con WorkBAT (por ejemplo, Driven; $r=0.14, p<0.01$ ), con DUWAS (por ejemplo, Work Excessive; $r=0.12, p<0.05$ ), con MBIGS (por ejemplo, Eficacia Personal; $r=0.35, p<$ 0.01). El Factor 2 correlaciona con el CPPC (por ejemplo, Potencial Creativo; $r=0.25, p<0.01$ ), con WorkBAT (por ejemplo, Work Enjoyment; $r=0.3, p<0.01$ ), con DUWAS (por ejemplo, Work Excessive; $r=0.17, p<0.05$ ) y con MBIGS (por ejemplo, Eficacia Personal; $r=0.37, p<$ 0.01). Y el tercer factor se asocia con el CPPC (por ejemplo, Potencial Creativo; $r=0.1, p<0.01$ ), con WorkBAT (por ejemplo, Work Enjoyment; $r=0.25, p<0.01$ ) y con MBI-GS (por ejemplo, Eficacia personal; $r=0.21, p<0.01$ ).

A modo de conclusión, se puede señalar que los análisis han aportado evidencia de la existencia de una estructura trifactorial, a la vez que muestran unos índices estadísticos adecuados (Tabachnick \& Fidell, 2007). La CEP es práctica y fácilmente administrable a empleados y a directivos. Asimismo, se posibilita una valoración independiente de cada una de ellas, mediante las tres subescalas que lo conforman. En cuanto a la aplicabilidad, ya que la escala presenta buenas propiedades psicométricas la información obtenida de la evaluación realizada a través del CEP puede permitir la implantación de programas de desarrollo de la creatividad (Leenders, van Engelen, \& Kratzer, 2007) en las organizaciones, con el fin de apoyar la creatividad y aminorar las barreras organizacionales que no permiten desarrollarla. Actualmente, las organizaciones modernas han de ser capaces de aprovechar el potencial creativo aletargado de los empleados para hacer frente a una amplia gama de retos y desafíos (DiLiello \& Houghton, 2006).

\section{Referencias}

Amabile, T. M. (1988). A model of creativity and innovation in organizations. En B. M. Staw \& L. L. Cummings (Eds.), Research in organizational behavior (Vol. 10, pp. 123-167).

Amabile, T. M. (1993). Motivational synergy: Toward a new conceptualization of intrinsic and extrinsic motivation in the workplace. Human Resource Management Review, 3, 185-201. http://dx.doi. org/10.1016/1053-4822(93)90012-S

Amabile, T. M. (1996). Creativity in context. Boulder, C: Westview Press.

Amabile, T. M. (1997). Motivating creativity in organizations: On doing what you love and loving what you do. California Management Review, 40(1), 39-58.

Amabile, T. M. (1998). How to kill creativity. Harvard Business Review, 76(5), 76-87.

Amabile, T. M., Hadley, C. N., \& Kramer, S. J. (2002). Creativity under the gun. Harvard Business Review, 80(8), 52-61.

Amabile, T. M., Schatzel, E. A., Moneta, G. B., \& Kramer, S. J. (2004). Leader behaviors and the work environment for creativity: Perceived leader support. The Leadership Quarterly, 15, 5-32. http:// dx.doi.org/10.1016/j.leaqua.2003.12.003

Balluerka, N., Gorostiaga, A., Alonso-Arbiol, I., \& Haranburu, M. (2007). La adaptación de instrumentos de medida de unas culturas a otras: una perspectiva práctica. Psicothema, 19(1), 124-133.

Bentler, P. M. (1990). Comparative fit indices in structural models. Psychological Bulletin, 107, 238-246. http://dx.doi.org/10.1037/0033-2909.107.2.238

Boada-Grau, J., De-Diego, R., De-Llanos-Serra, E., \& Vigil-Colet, A. (2011). Short Spanish version of Team Climate Inventory (TCI-14): Development and psychometric properties. Psicothema, 23(2), 308-313.

Boada-Grau, J., Prizmic-Kuzmica, A. -J., González-Fernández, M. D., \& Vigil-Colet, A. (2013). Spanish version of Bus Drivers' Job Demands Scale. Psicothema, 25(2), 258-265. http://dx.doi.org/10.7334/ psicothema2012.106

Boada-Grau, J., Prizmic-Kuzmica, A. -J., Serrano-Fernández, M. J., \& Vigil-Colet, A. (en prensa). Es- 
tructura factorial, fiabilidad y validez de la escala de adicción al trabajo (WorkBAT): versión española. Anales de Psicología.

Boada-Grau, J., Sánchez-García, J. C., Prizmic-Kuzmica, A. -J., \& Vigil-Colet, A. (2012). Work health and hygiene in the transport industry (TRANS-18): Factorial structure, reliability and validity. The Spanish Journal of Psychology, 15(1), 357-366. http:// dx.doi.org/10.5209/rev_SJOP.2012.v15.n1.37342

Boada-Grau, J., Sánchez-García, J. C., Prizmic-Kuzmica, A. -J., \& Vigil-Colet, A. (2013). Adaptación de la escala de medida sobre el Potencial Creativo y Creatividad Práctica (PCCP-17). Manuscrito inédito, Universitat Rovira i Virgili, Tarragona, España.

Bryant, S. E. (2003). The role of transformational and transactional leadership in creating, sharing and exploiting organizational knowledge. Journal of Leadership and Organizational Studies, 9(4), 32-44. http://dx.doi.org/10.1177/107179190300900403

Burke, R. J., Richardsen, A. M., \& Martinussen, M. (2002). Psychometric properties of Spence and Robbins' measures of workaholism components. Psychological Reports, 91(3f), 1098-1104. http:// dx.doi.org/10.2466/pr0.2002.91.3f.1098

De Dreu, C. K. W., Baas, M., \& Nijstad, B. A. (2008). Hedonic tone and activation in the mood-creativity link: Towards a dual pathway to creativity model. Journal of Personality and Social Psychology, 94(5), 739-756. http://dx.doi.org/10.1037/00223514.94.5.739

Del-Líbano, M., Llorens, S., Salanova, M., \& Schaufeli, W. (2010). Validity of a brief workaholism scale. Psicothema, 22(1), 143-150.

DiLiello, T. C., \& Houghton, J. D. (2006). Maximizing organizational leadership capacity for the future: Toward a model of self-leadership, innovation and creativity. Journal of Managerial Psychology, 21(4), 319-337. http://dx.doi. org/10.1108/02683940610663114

DiLiello, C., \& Houghton, J. D. (2008). Creative potential and practiced creativity: Identifying untapped creativity in organizations. Creativity and Innovation Management, 17(1), 37-46. http://dx.doi. org/10.1111/j.1467-8691.2007.00464.x
Fan, X., \& Sivo, S. A. (2007). Sensitivity of fit indices to model misspecification and model types. Multivariate Behavioral Research, 42(3), 509-529.

Forbes, J. B., \& Domm, D. R. (2004). Creativity and productivity: Resolving the conflict. SAM Advanced Management Journal, 69(2), 4-13.

Hambleton, R. K., Merenda, P. F., \& Spielberger, C. D. (2005). Adapting educational and psychological tests for cross-cultural assessment. London: Erlbaum.

Hu, L., \& Bentler, P. (1999). Cutoff criteria for fit indexes in covariance structure analysis: Conventional criteria versus new alternatives. Structural Equation Modeling, 6(1), 1-55.

Joreskog, K. G. (1993). Testing structural equation models. En K. A. Bollen \& J. S. Long (Eds.), Testing structural equation models (pp. 294-316). Newbury Park, CA: Sage.

Kerlinger, F. N. (2001). Investigación del comportamiento: métodos de investigación en ciencias sociales. México: McGraw-Hill.

Leenders, R., Van Engelen, J., \& Kratzer, J. (2007). Systematic design methods and the creative performance of new product teams: Do they contradict or complement each other? Journal of Product Innovation and Management, 24(2), 166-179. http:// dx.doi.org/10.1111/j.1540-5885.2007.00241.x

Lévy-Mangin, J. P., \& Varela-Mallou, J. (2006). Modelización con estructuras de covarianzas en ciencias sociales. Temas esenciales, avanzados y aportaciones especiales. Madrid: Gesbiblo.

Mayfield, J., \& Mayfield, M. (2005). The effects of leader motivating language use on worker perceptions of the creative environment. Decision Sciences Institute.

Mayfield, J., \& Mayfield, M. (2007). The effects of leader communication on worker's intent to stay: An investigation using structural equation modeling. Human Performance, 20(2), 85-102. http://dx.doi. org/10.1080/08959280701332018

Mayfield, J., \& Mayfield, M. (2008). The creative environment's influence on intent to turnover: A structural equation model and analysis. Management Research News, 31(1), 41-56. http://dx.doi. org/10.1108/01409170810845949

Mayfield, M., \& Mayfield, J. (2010). Developing a scale to measure the creative environment perceptions: A questionnaire for investigating garden variety 
creativity. Creativity Research Journal, 22(2), 162 169. http://dx.doi.org/10.1080/10400419.2010.4 81511

McLean, L. D. (2005). Organizational culture's influence on creativity and innovation: A review of the literature and implications for human resource development. Advances in Developing Human Resources, 7(2), 226-246. http://dx.doi. org/10.1177/1523422305274528

McMillan, L. H. W., Brady, E. C., O'Driscoll, M. P., \& Marsh, N. V. (2002). A multifaceted validation study of Spence and Robbins' (1992) Workaholism Battery. Journal of Occupational and Organizational Psychology, 75(3), 357-368. http://dx.doi. org/10.1348/096317902320369758

Mueller, J. S., Melwani, S., \& Goncalo, A. (2012). The bias against creativity: Why people desire but reject creative ideas. Psychological Science January, 23(1), 13-17. http://dx.doi.org/10.1177/0956797611421018

Mumford, D. (2012). Handbook of organizational creativity. Amsterdam: Elsevier/Academic Press.

Mumford, M. D., Scott, G. M., Gaddis, B., \& Strange, J. M. (2002). Leading creative people: Orchestrating expertise and relationships. The Leadership Quarterly, 13(6), 705-750. http://dx.doi.org/10.1016/ S1048-9843(02)00158-3

Muñiz, J., \& Bartram, D. (2007). Improving international tests and testing. European Psychologist, 12(3), 206-219. http://dx.doi.org/10.1027/10169040.12.3.206

Salanova, M., Schaufeli, W. B., Llorens, S., Peiró, J. M., \& Grau, R. (2000). Desde el 'burnout' al 'engagement': iuna nueva perspectiva? Revista de Psicología del Trabajo y las Organizaciones, 16(2), 117-134.
Schaufeli, W. B., Leiter, M. P., Maslach, C., \& Jackson, S. E. (1996). Maslach Burnout Inventory - General Survey. En C. Maslach, S. E. Jackson \& M. P. Leiter (Eds.), The Maslach Burnout Inventory-Test Manual (3.a ed., pp. 22-26). Palo Alto, CA: Consulting Psychologists Press.

Schaufeli, W. B., Shimazu, A., \& Taris, T. W. (2009). Driven to work excessively hard: The evaluation of a two-factor measure of workaholism in the Netherlands and Japan. Cross-Cultural Research, 43(4), 320 348. http://dx.doi.org/10.1177/1069397109337239

Schumacker, R. E., \& Lomax, R. G. (1996). A beginner's guide to structural equation modeling. Mahwah, NJ: Erlbaum.

Shalley, C. E., Gilson, L. L., \& Blum, T. C. (2000). Matching creativity requirements and the work environment: Effects on satisfaction and intentions to leave. Academy of Management Journal, 43(2), 215-223. http://dx.doi.org/10.2307/1556378

Stoker, J. I., Looise, J. C., Fisscher, O. A. M., \& De Jong, R. D. (2001). Leadership and innovation: Relations between leadership, individual characteristics and the functioning of $R \& D$ teams. The International Journal of Human Resource Management, 12(7), 1141-1151.

Tabachnick, B. G., \& Fidell, L. S. (2007). Using multivariate statistic. Boston: Allyn \& Bacon.

Von Krogh, G. (1998). Care in knowledge creation. California Management Review, 40(3), 133-153. http:// dx.doi.org/10.2307/41165947

Williams, S. (2001). Increasing employees' creativity by training their managers. Industrial and Commercial Training, 33(2), 63-68. http://dx.doi. org/10.1108/00197850110385642 


\section{Apéndice}

Versión española de la escala CEP-9

Instrucciones: A continuación encontrarás algunas afirmaciones sobre TU TRABAJO Y TU ORGANIZACIÓN. No hay respuestas correctas ni incorrectas, ni tampoco respuestas buenas o malas. Lee atentamente cada frase e indica por favor el grado de desacuerdo o acuerdo con respecto a las mismas.

\begin{tabular}{|c|c|c|c|c|}
\hline 1 & 2 & 3 & 4 & 5 \\
$\begin{array}{c}\text { Completamente } \\
\text { en desacuerdo }\end{array}$ & En desacuerdo & Neutral & De acuerdo & $\begin{array}{c}\text { Completamente } \\
\text { de acuerdo }\end{array}$ \\
\hline
\end{tabular}

\begin{tabular}{|c|c|c|c|c|c|}
\hline \multirow{2}{*}{$\begin{array}{l}\text { 1.- Mi superior me anima a ser creativo/a [My supervisor encourages me to be } \\
\text { creative]. }\end{array}$} & 1 & 2 & 3 & 4 & 5 \\
\hline & & & & & \\
\hline $\begin{array}{l}\text { 2.-Mi grupo de trabajo apoya las nuevas maneras de hacer las cosas [My work } \\
\text { group is supportive of new ways of doing things]. }\end{array}$ & & & & & \\
\hline $\begin{array}{l}\text { 3.-Mi organización me anima a trabajar de forma creativa [My organization } \\
\text { encourages me to work creatively]. }\end{array}$ & & & & & \\
\hline $\begin{array}{l}\text { 4.- Cuento con los recursos necesarios para llevar a cabo mi trabajo [I have the } \\
\text { resources I need to do my job]. }\end{array}$ & & & & & \\
\hline 5.-Mi trabajo es retador [My work is challenging]. & & & & & \\
\hline $\begin{array}{l}\text { 6.-Tengo control sobre cómo hago mi trabajo [I have control over how I do my } \\
\text { work]. }\end{array}$ & & & & & \\
\hline $\begin{array}{l}\text { 7.-La política de mi organización dificulta la creatividad [My organization's } \\
\text { politics makes it difficult to be creative]. }\end{array}$ & & & & & \\
\hline $\begin{array}{l}\text { 8.-Las políticas de mi organización impiden la espontaneidad en el trabajo [My } \\
\text { organization's policies impedes spontaneity in the workplace] }\end{array}$ & & & & & \\
\hline $\begin{array}{l}\text { 9.-Es difícil ser creativo con los plazos con los que trabajo [It is difficult to be } \\
\text { creative with the work deadlines I have]. }\end{array}$ & & & & & \\
\hline
\end{tabular}

